\title{
A SPECIAL SUBLATTICE OF THE CONGRUENCE LATTICE OF A REGULAR SEMIGROUP
}

\author{
by MARIO PETRICH
}

(Received 26th May 1995)

Dedicated to the memory of Professor Umberto Gasapina

\begin{abstract}
Let $S$ be a regular semigroup and $\mathscr{C}(S)$ be its congruence lattice. For $\rho \in \mathscr{C}(S)$, we consider the sublattice $L_{\rho}$ of $\mathscr{C}(S)$ generated by the congruences $\rho w$ where $w \in\{K, k, T, t\}^{*}$ and $w$ has no subword of the form $K T$, $T K, k t, t k$. Here $K, k, T, t$ are the operators on $\mathscr{C}(S)$ induced by the kernel and the trace relations on $\mathscr{C}(S)$. We find explicitly the least lattice $L$ whose homomorphic image is $L_{\rho}$ for all $\rho \in \mathscr{B}(S)$ and represent it as a distributive lattice in terms of generators and relations. We also consider special cases: bands of groups, $E$ unitary regular semigroups, completely simple semigroups, rectangular groups as well as varieties of completely regular semigroups.
\end{abstract}

1991 Mathematics subject classification: Primary 20M10; Secondary $20 \mathrm{M} 17$.

\section{Introduction and summary}

Congruences on a regular semigroup have recently attracted considerable attention which they richly deserve. For they exhibit various features intimately related to the structure of the semigroup. The emergence of the kernel-trace approach provided additional impetus whose full effect has most probably not reached its climax yet.

For a regular semigroup $S$ and its congruence $\rho$, it is natural to consider the lattice $L_{\rho}$ generated by the set of congruences obtained from $\rho$ by the repeated application of the operators

$$
K: \lambda \longrightarrow \lambda^{K}, k: \lambda \longrightarrow \lambda_{K}, T: \lambda \longrightarrow \lambda^{T}, t: \lambda \longrightarrow \lambda_{T}
$$

Here $\lambda^{K}, \lambda_{K}, \lambda^{T}, \lambda_{T}$ denote, respectively, the greatest and the least congruences on $S$ with the same kernel and trace as $\lambda$. For an arbitrary congruence $\rho$ on $S$, it appears difficult to characterize this lattice.

We may limit our scope in various ways in order to arrive at some more tractable problems. In particular $(\alpha)$ we may require that $S$ satisfy suitable conditions bearing upon the behaviour of congruences and/or $(\beta)$ we may consider the lattice generated only by a small subset of the above set.

As the restriction of type $(\alpha)$, essential to all our discussion, we require that the kernel relation $\mathscr{K}$ on the lattice $\mathscr{C}(S)$ of congruences on $S$ be a congruence. This is a 
strong restriction indeed, but we point out important examples of regular semigroups with this property. For example, primitive regular semigroups have this property ([6, Theorem 3.6]). For strong semilattices of simple regular semigroups, for Reilly semigroups and for a retract extension of one Brandt semigroup by another, we gave necessary and sufficient conditions for the kernel relation to be a congruence ([6, Theorems 4.3, 4.7, 5.5] and [5, Theorem 5.7]).

For the restriction of type $(\beta)$ above, we have chosen the sublattice of the following description. With the notation of (1), we consider the lattice generated by the set

$\left\{\rho w \mid w \in\{K, k, T, t\}^{*}, w\right.$ has no subword of the form $\left.K T, T K, k t, t k\right\}$,

where ()* means the free monoid.

For fully invariant congruences on a free completely regular semigroup $F \mathscr{C} \mathscr{R}$ of countably infinite rank, Pastijn-Trotter ([4, Theorem 5.3]) determined the lattice generated by the set

$$
\left\{\rho w \mid w \in\{K, k, T, t\}^{*}, w \text { has no subword of the form } K t, t K, k T, T k\right\},
$$

If $\rho$ is a fully invariant congruence on $F \mathscr{C} \mathscr{R}$, then $\rho K, \rho k, \rho T, \rho t$ are all fully invariant and hence the case studied in [4] restricts only $\rho$ to be fully invariant and $S$ to be equal to $F \mathscr{C} \mathscr{R}$. Pastijn ([2, Theorem 11]) proved that the kernel relation restricted to fully invariant congruences on $F \mathscr{C} \mathscr{R}$ is a congruence. Hence in this case, we again have the restriction $(\alpha)$ above, whereas the restriction of type $(\beta)$ adopted in [4] seems to be, in a certain sense, diametrically opposite to ours.

Section 2 contains most of the needed preliminaries. A sequence of lemmas in Section 3 leads to the main result of the paper which characterizes our lattice as a homomorphic image of a lattice given by a diagram. We study in Section 4 certain conditions on a regular semigroup which imply that some of the vertices of our lattice coincide. In Section 5 we restrict our attention to completely simple semigroups where we also present an example.

\section{Preliminaries}

We generally follow the notation and terminology of [1]. We list only a few of the most frequently used notations.

Throughout the paper $S$ denotes a regular semigroup, $E(S)$ denotes the set of its idempotents and $\mathscr{C}(S)$ the lattice of its congruences. For $\rho \in \mathscr{C}(S)$,

$$
\begin{aligned}
& \operatorname{ker} \rho=\{a \in S \mid a \rho e \text { for some } e \in E(S)\}, \\
& \operatorname{tr} \rho=\left.\rho\right|_{E(S)}
\end{aligned}
$$

are, respectively, the kernel and the trace of $\rho$. Relations $\mathscr{H}$ and $\mathscr{T}$ are defined on $\mathscr{C}(S)$ by 


$$
\lambda \mathscr{K} \rho \Leftrightarrow \operatorname{ker} \lambda=\operatorname{ker} \rho, \quad \lambda \mathscr{T} \rho \Leftrightarrow \operatorname{tr} \lambda=\operatorname{tr} \rho .
$$

We call $\mathscr{K}$ the kernel relation for $S$. For any $\rho \in \mathscr{C}(S)$, we denote by

$\rho K, \rho k$ - the greatest and the least congruences on $S$ with the same kernel as $\rho$, respectively,

$\rho T, \rho t$ - the greatest and least congruences on $S$ with the same trace as $\rho$, respectively,

and consider $K, k, T$ and $t$ as operators on $\mathscr{C}(S)$.

For any set $X$, we denote by $X^{*}$ the free monoid on $X, \mathscr{F} \mathscr{D} \mathscr{L}(X)$ the free distributive lattice on $X$, and $\epsilon$ and $\omega$ the equality and universal relations on $X$. If $\rho$ is a relation on $S, \rho^{*}$ denotes the congruence on $S$ generated by $\rho$.

We will use freely the well-known results that $\mathscr{K}$ is a $\wedge$-congruence and $\mathscr{T}$ is a congruence on $\mathscr{C}(S)$, as well as that $\mathscr{K} \wedge \mathscr{T}=\epsilon$. For proofs of these statements, see ([3, Lemma 2.5(i), Corollaries 4.9 and 2.11]).

\section{The theorem}

Five lemmas will be needed for the proof of this theorem certain of which provide additional information.

Lemma 3.1. Let $\lambda, \rho \in \mathscr{C}(S)$. If $\lambda \subseteq \rho$, then $\lambda k \subseteq \rho k, \lambda T \subseteq \rho T, \lambda t \subseteq \rho t$.

Proof. Assume that $\lambda \subseteq \rho$. Then $\lambda k \wedge \rho k \mathscr{K} \lambda \wedge \rho=\lambda$ since $\mathscr{K}$ is a $\wedge$-congruence, and thus $\lambda k \subseteq \lambda k \wedge \rho k$ and finally $\lambda k \subseteq \rho k$. Further, $\lambda T \vee \rho T \mathscr{T} \lambda \vee \rho=\rho$ since $\mathscr{T}$ is a congruence whence $\lambda T \vee \rho T \subseteq \rho T$ and thus $\lambda T \subseteq \rho T$. The relation $\lambda t \subseteq \rho t$ follows as for $\lambda k \subseteq \rho k$ using the congruence $\mathscr{T}$.

Lemma 3.2. The following conditions are equivalent.

(i) $\mathscr{K}$ is a congruence on $\mathscr{C}(S)$.

(ii) For any $\lambda, \rho \in \mathscr{C}(S), \lambda \subseteq \rho$ implies $\lambda K \subseteq \rho K$.

(iii) For any $\lambda, \rho \in \mathscr{C}(S), \lambda \vee \rho \mathscr{H} \lambda K \vee \rho$. $T$.

Proof. (i) $\rightarrow$ (ii). The argument here is the same as in the proof of Lemma 3.1 for

(ii) $\rightarrow$ (iii). Let $\lambda, \rho \in \mathscr{C}(S)$. Then $\lambda \vee \rho \subseteq \lambda K \vee \rho$ and hence $(\lambda \vee \rho) K \subseteq(\lambda K \vee \rho) K$. Also $\lambda \subseteq \lambda \vee \rho$ implies $\lambda K \subseteq(\lambda \vee \rho) K$ and $\rho \subseteq(\lambda \vee \rho) K$ whence $\lambda K \vee \rho \subseteq(\lambda \vee \rho) K$ so that $(\lambda K \vee \rho) K \subseteq(\lambda \vee \rho) K$ and equality prevails.

(iii) $\rightarrow$ (i). Let $\lambda, \rho, \theta \in \mathscr{C}(S)$ and assume that $\lambda \mathscr{K} \rho$. Then $\lambda K=\rho K$ and thus

$$
\lambda \vee \theta \mathscr{K} \lambda K \vee \theta=\rho K \vee \theta \mathscr{K} \rho \vee \theta .
$$


Lemma 3.3. The following statements are valid: $T k T=T k, k T k=k T$. If $\mathscr{K}$ is a congruence, then also: $K t K=K t, t K t=t K$.

Proof. We will use freely Lemma 3.1. Let $\rho \in \mathscr{C}(S)$. First $\rho T k T \subseteq \rho T T=\rho T$; then $k e r \rho T k T \supseteq k e r \rho T k=k e r \rho T$ and thus $\operatorname{ker} \rho T k T=k e r \rho T=k e r \rho T k$. Also $\operatorname{tr} \rho T k T=\operatorname{tr} \rho T k$ and therefore $\rho T k T=\rho T k$.

Further $\rho k T k \supseteq \rho k k=\rho k$; next $\operatorname{tr} \rho k T k \subseteq \operatorname{tr} \rho k T=\operatorname{tr} \rho k$ and hence $\operatorname{tr} \rho k T k=$ $\operatorname{tr} \rho k=\operatorname{tr} \rho k T$. Also ker $\rho k T k=k e r \rho k T$ and therefore $k T k=k T$.

If $\mathscr{K}$ is a congruence, we may use both Lemmas 3.1 and 3.2 , and by interchanging the roles of $t$ and $k$, capital and lower case, above obtain the remaining two equalities.

Lemma 3.4. Let $\mathscr{K}$ be a congruence and let $\rho \in \mathscr{C}(S)$.

(i) $\operatorname{tr} \rho k \subseteq \operatorname{tr} \rho T k \subseteq \operatorname{tr} \rho \subseteq \operatorname{tr} \rho t K \subseteq \operatorname{tr} \rho K$.

(ii) $k e r \rho t \subseteq k e r \rho K t \subseteq k e r \rho \subseteq k e r \rho k T \subseteq k e r \rho T$.

Proof. We will freely use Lemmas 3.1 and 3.2 .

(i) First $\rho \subseteq \rho T$ implies $\rho k \subseteq \rho T k$ and thus $\operatorname{tr} \rho k \subseteq \operatorname{tr} \rho T k$. Now let $e, f \in E(S)$. If $e \rho T k f$, then $e \rho T f$ and so $e \rho f$ which proves that $\operatorname{tr} \rho T k \subseteq \operatorname{tr} \rho$. If $e \rho f$, then $e \rho t f$ so also $e \rho t K f$ which proves that $\operatorname{tr} \rho \subseteq \operatorname{tr} \rho t K$. Since $\rho t \subseteq \rho$, we have $\rho t K \subseteq \rho K$ and thus $\operatorname{tr} \rho t K \subseteq \operatorname{tr} \rho K$.

(ii) If $a \in k e r \rho K t$, then $a \rho K t e$ for some $e \in E(S)$ whence $a \rho K e$ and thus $a \in k e r \rho K=k e r \rho$. Hence ker $\rho K t \subseteq k e r \rho$. If $a \in k e r \rho$, then $a \in k e r \rho k \subseteq k e r \rho k T$. Therefore ker $\rho \subseteq k e r \rho k T$. The remaining two inclusions follow by monotonicity, as above.

Lemma 3.5. Let $\lambda, \rho \in \mathscr{C}(S)$.

(i) $\operatorname{tr} \lambda \subseteq \operatorname{tr} \rho \Leftrightarrow \operatorname{tr}(\lambda \wedge \rho)=\operatorname{tr} \lambda \Leftrightarrow \operatorname{tr}(\lambda \vee \rho)=\operatorname{tr} \rho$.

(ii) $\operatorname{ker} \lambda \subseteq \operatorname{ker} \rho \Leftrightarrow \operatorname{ker}(\lambda \wedge \rho)=\operatorname{ker} \lambda$.

(iii) If $\mathscr{K}$ is a congruence, then $\operatorname{ker} \lambda \subseteq \operatorname{ker} \rho \Leftrightarrow \operatorname{ker}(\lambda \vee \rho)=\operatorname{ker} \rho$.

Proof. Straightforward from the congruence properties of $\mathscr{T}$ and $\mathscr{K}$.

We are now ready for the principal result of the paper.

Theorem 3.6. Let $S$ be a regular semigroup for which $\mathscr{K}$ is a congruence. Fix $\rho \in \mathscr{C}(S)$ and let $L_{\rho}$ be the sublattice of $\mathscr{C}(S)$ generated by the set

$$
\left\{\rho w \mid w \in\{K, k, T, t\}^{*}, w \text { has no subword of the form } K T, T K, k t, t k\right\} .
$$

Then $L_{\rho}$ is a homomorphic image of the lattice $L$ depicted by the following diagram. 


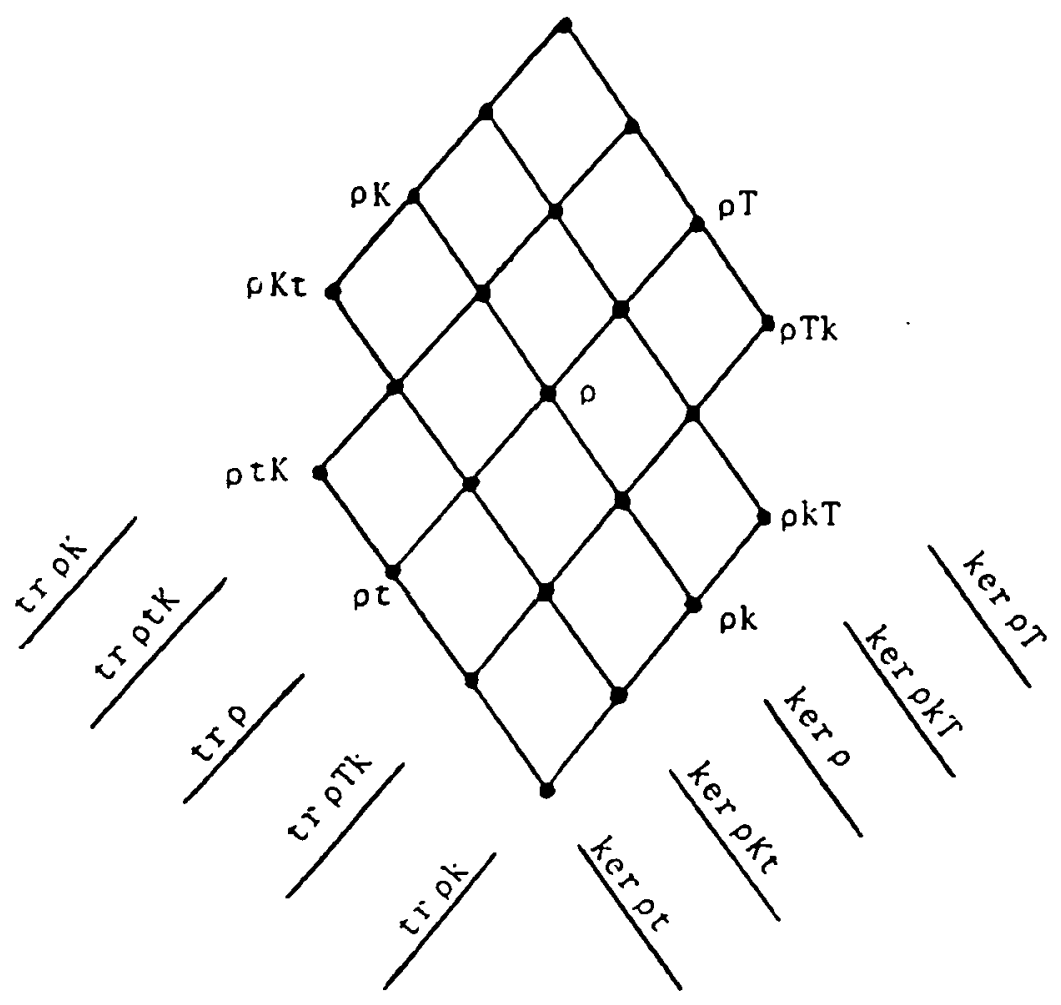

Labels on the sides indicate traces and kernels. Moreover,

$$
L \cong \mathscr{F} \mathscr{D} \mathscr{L}(\rho t K, \rho K t, \rho, \rho k T, \rho T k) / \mathscr{R}
$$

where

$$
\mathscr{R}=(\rho t K \leq \rho K t, \rho k T \leq \rho T k, \rho K t \wedge \rho T k \leq \rho \leq \rho t K \vee \rho k T)
$$

and none of these relations may be omitted.

Proof. First Lemma 3.3 asserts that the set in (2) equals

$$
\{\rho, \rho K, \rho k, \rho T, \rho t, \rho K t, \rho k T, \rho T k, \rho t K\} \text {. }
$$

Hence we must characterize the lattice generated by the set (3). Starting with $\rho$ and applying the formula

$$
\rho K \wedge \rho T=\rho=\rho k \vee \rho t
$$


see ([3, Theorem 3.5]), and applying the operators $K, k, T, t$ to the resulting congruences, we obtain the part of the above diagram consisting of the congruences listed in (3) and representing a partially ordered set. Plotting these vertices in the diagram, we draw next the lines indicating the $\mathscr{K}$ - and $\mathscr{T}$-classes of these vertices observing that $\rho k, \rho$ and $\rho K$ are $\mathscr{K}$-related and $\rho t, \rho$ and $\rho T$ are $\mathscr{T}$-related.

The resulting $\mathscr{K}$ - as well as $\mathscr{T}$-classes are linearly ordered as established in Lemma 3.4. We can now identify the joins and the meets of congruences in (3) by the intersections of the corresponding $\mathscr{K}$ - and $\mathscr{T}$-classes following the rule in Lemma 3.5. In order to see that $L_{\rho}$ is a homomorphic image of the lattice $L$, we first coordinatize $L$ as follows. We label the lines in the left hand corner of its diagram by the numbers $1,2,3,4,5$ from the bottom to the top, and do the same for the lines in the right hand corner. Let $I=\{1,2,3,4,5\}$. Hence the lattice $L$ may be coordinatized by the set

$$
T=(I \times I) \backslash\{(1,5),(5,1)\}
$$

with componentwise usual order of integers. Suppose that $L_{\rho}$ satisfies a relation $u=v$ which corresponds to $(i, j) \sim(k, l)$. Call the sets

$$
\{(x, y) \mid y \in I\}, \quad\{(x, y) \mid x \in I\}
$$

the rows and the columns of $L$, respectively. By taking meets and joins with suitable elements, we see that $\sim$ induces the congruence on $L$ which identifies all the rows between $i \wedge k$ and $i \vee k$ and all the columns between $j \wedge l$ and $j \vee l$. If we do this for every relation $u=v$ valid in $L_{\rho}$, we obtain a congruence $\theta$ on $L$ with the property that $L / \theta \cong L_{\rho}$. It follows that for our particular regular semigroup $S$ and the particular congruence $\rho$, the lattice $L_{\rho}$ is a homomorphic image of the lattice depicted by the above diagram.

We prove next the isomorphism assertion of the theorem. It will be convenient to introduce the notation

$$
a=\rho t K, \quad A=\rho K t, \quad b=\rho k T, \quad B=\rho T k .
$$

Our task is to construct the free distributive lattice $D$ on the generators $a, A, b, B, \rho$ subject to the relations

$$
a \leq A, \quad b \leq B, \quad A \wedge B \leq \rho \leq a \vee b .
$$

We consider $D$ as a subdirect product of copies of the nontrivial subdirectly irreducible distributive lattice $Y=\{0,1\}$. Since $D$ is generated by five elements, homomorphisms of $D$ into $Y$ may be represented by all quintuples of 0 's and 1's which satisfy the restrictions (5): 


\begin{tabular}{ccccc}
$a$ & $A$ & $b$ & $B$ & $\rho$ \\
\hline 1 & 1 & 0 & 0 & 0 \\
1 & 1 & 0 & 0 & 1 \\
1 & 1 & 0 & 1 & 1 \\
\hline 0 & 0 & 1 & 1 & 0 \\
0 & 0 & 1 & 1 & 1 \\
0 & 1 & 1 & 1 & 1 \\
\hline 0 & 0 & 0 & 1 & 0 \\
0 & 1 & 0 & 0 & 0
\end{tabular}

where we have omitted the quintuples consisting entirely of 0's or entirely of 1's above since they provide no information.

We wish to represent the elements of $D$ as joins of meets of generators (which is possible since $D$ is distributive). We obtain

$$
\begin{array}{ll}
a=(11100000) & a \wedge b=(00000000) \\
A=(11100101) & a \wedge B=(00100000) \\
b=(00011100) & a \wedge \rho=(01100000) \\
B=(00111110) & \\
\rho=(01101100) & b \wedge \rho=(00001100) \\
A \wedge b=(00000100) & B \wedge \rho=(00101100) \\
A \wedge B=(00100100) &
\end{array}
$$

and the remaining meets equal one of those above. Now

$$
\begin{aligned}
& a \vee b=(11111100) \\
& a \vee B=(11111110) \\
& a \vee \rho=(11101100) \\
& b \vee \rho=(01111100) \\
& B \vee \rho=(01111110)
\end{aligned}
$$

$$
\begin{aligned}
& A \vee b=(11111101) \\
& A \vee B=(11111111) \\
& A \vee \rho=(11101101) \\
& a \vee(A \wedge b)=(11100100) \\
& b \vee(a \wedge B)=(00111100)
\end{aligned}
$$

and the remaining joins of meets equal one of those above. Instead of checking the last two assertions, we may draw the diagram with the above octuples as vertices and verify that they already form a lattice. Now taking into account the change of notation (4), we see that Diagram 1 is essentially identical with the one in the statement of the theorem, which proves the isomorphism assertion.

In order to prove the last claim of the theorem, we construct quintuples each of which satisfies all the restrictions in (5) except one as follows. 


\begin{tabular}{r|ccccc} 
& $a$ & $A$ & $b$ & $B$ & $\rho$ \\
\hline$a \leq A$ & 1 & 0 & 0 & 0 & 1 \\
$b \leq B$ & 0 & 0 & 1 & 0 & 1 \\
$A \wedge B \leq \rho$ & 0 & 1 & 0 & 1 & 0 \\
$\rho \leq a \vee b$ & 0 & 0 & 0 & 0 & 1
\end{tabular}

Example 3.7. Let $S$ be the semigroup of all transformations on the set $\{1,2\}$, say

$$
\alpha=\left(\begin{array}{ll}
1 & 2 \\
1 & 2
\end{array}\right), \quad \beta=\left(\begin{array}{ll}
1 & 2 \\
2 & 1
\end{array}\right), \quad \gamma=\left(\begin{array}{ll}
1 & 2 \\
1 & 1
\end{array}\right), \quad \delta=\left(\begin{array}{ll}
1 & 2 \\
2 & 2
\end{array}\right) .
$$

Let $\rho$ be the Rees congruence relative to the minimal ideal. Then $\rho T=\rho T k=\eta$, the least semilattice congruence on $S$, and $\rho k=\rho k T=\epsilon$. Therefore $\operatorname{tr} \rho k \subset \operatorname{tr} \rho T k$ and ker $\rho k T \subset \operatorname{ker} \rho T$. Also, $\mathscr{C}(S)$ is a chain with $\mathscr{K}$-classes: $\{\epsilon, \rho\},\{\eta, \omega\}$ whence it follows easily that $\mathscr{K}$ is a congruence.

The above example shows that in the diagram of Theorem 3.6, the $\mathscr{T}$-classes of $\rho k$ and $\rho T k$ are distinct and the $\mathscr{K}$-classes of $\rho k T$ and $\rho T$ are also distinct. We will see in Example 5.4 that the remaining kernel and trace classes are in general also distinct. This implies that no proper homomorphic image of the lattice depicted in Theorem 3.6 has the property enunciated in that theorem.

We recall from Section 1 the case considered by Pastijn-Trotter [4]. As in the case they studied, we could consider, for an arbitrary regular semigroup $S$, a sublattice $L$ of $\mathscr{C}(S)$ for which $\left.\mathscr{K}\right|_{L}$ is a congruence. The above arguments would go through in this case with obvious modifications. Hence letting $L$ be the lattice of fully invariant congruences on $F \mathscr{C} \mathscr{R}$, we obtain a result concerning varieties of completely regular semigroups. For the operators $K, k, T$ and $t$ may be transferred to the lattice $\mathscr{L}(\mathscr{C} \mathscr{R})$ of varieties of completely regular semigroups via the usual antiisomorphisms

$$
\rho \rightarrow[\rho], \quad \mathscr{r} \longrightarrow \rho_{\mathscr{r}}
$$

of $L$ and $\mathscr{L}(\mathscr{C} \mathscr{R})$. Indeed, for any $\mathscr{V} \in \mathscr{L}(\mathscr{C} \mathscr{R})$, we set

$$
\mathscr{V} K=\left[\rho_{\mathscr{V}} k\right], \quad \mathscr{V} k=\left[\rho_{\mathscr{V}} K\right], \quad \mathscr{V} T=\left[\rho_{\mathscr{r}} t\right], \quad \mathscr{V} t=\left[\rho_{\mathscr{V}} T\right] .
$$

With this preamble, Theorem 3.6 has the following consequence.

Corollary 3.8. Fix $\mathscr{V} \in \mathscr{L}(\mathscr{C} \mathscr{R})$ and let $L_{\mathscr{V}}$ be the lattice generated by the set $\left\{\mathscr{V} w \mid w \in\{K, k, T, t\}^{*}, w\right.$ has no subword of the form $\left.K T, T K, k t, t k\right\}$.

Then $L_{r}$ is a homomorphic image of the lattice depicted by the diagram 


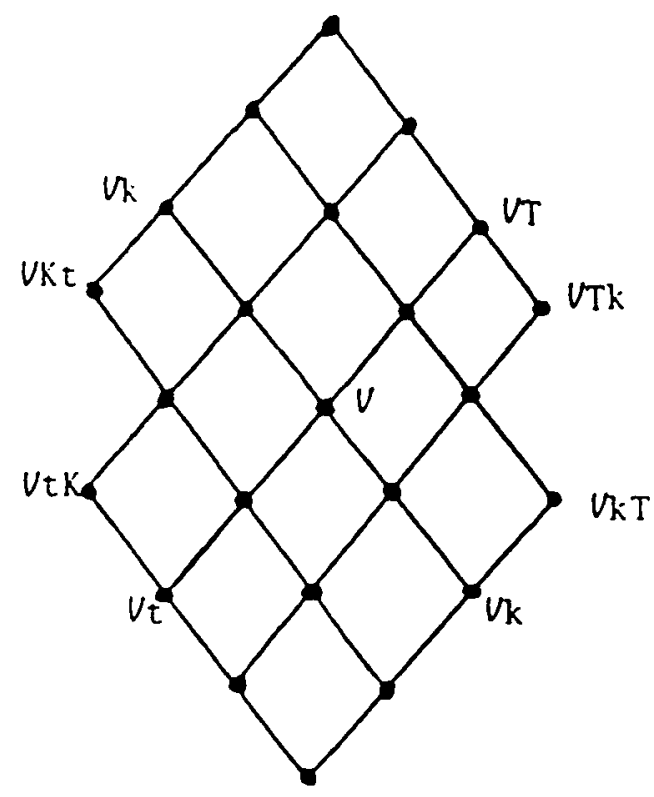

\section{Two special cases}

We consider here the cases when $S$ is a band of groups and when $k e r \sigma=E(S)$ ( $E$-unitary regular semigroups).

Proposition 4.1. The following conditions on a completely regular semigroup $S$ are equivalent.

(i) $\mathscr{H}=\mathscr{H}^{*}$.

(ii) For every $\rho \in \mathscr{C}(S), \rho k=\rho \wedge \mathscr{H}$.

(iii) For every $\rho \in \mathscr{C}(S), \rho T=\rho \vee \mathscr{H}$ (join of equivalence relations).

(iv) For every $\rho \in \mathscr{C}(S), \rho k T=\mathscr{H}$.

(v) For every $\rho \in \mathscr{C}(S), \rho T k=\mathscr{H}$.

Proof. Let $\rho$ stand for any congruence on $S$.

(i) $\rightarrow$ (ii). Indeed

$$
\begin{aligned}
& \operatorname{ker}(\rho \wedge \mathscr{H})=\operatorname{ker} \rho \wedge \text { ker } \mathscr{H}=\text { ker } \rho \cap S=\text { ker } \rho, \\
& \operatorname{tr}(\rho \wedge \mathscr{H})=\operatorname{tr} \rho \wedge \operatorname{tr} \mathscr{H}=\operatorname{tr} \rho \wedge \epsilon=\epsilon,
\end{aligned}
$$

which evidently implies that $\rho k=\rho \wedge \mathscr{H}$. 
(i) $\rightarrow$ (iii). Indeed, $\operatorname{ker}(\rho \vee \mathscr{H})=\operatorname{ker} \mathscr{H}=S$ and

$$
\operatorname{tr}(\rho \vee \mathscr{H})=\operatorname{tr} \rho \vee \operatorname{tr} \mathscr{H}=\operatorname{tr} \rho \vee \epsilon=\operatorname{tr} \rho
$$

which evidently implies that $\rho T=\rho \vee \mathscr{H}$.

Now assuming (i) and using (ii) and (iii), we obtain

$$
\rho k T=(\rho \wedge \mathscr{H}) \vee \mathscr{H}=\mathscr{H}, \quad \rho T k=(\rho \vee \mathscr{H}) \wedge \mathscr{H}=\mathscr{H},
$$

giving (iv) and (v). If (ii) holds, then $\mathscr{H}=\omega \wedge \mathscr{H}=\omega k \in \mathscr{C}(S)$. If (iii) holds, then $\mathscr{H}=\epsilon \vee \mathscr{H}=\epsilon T \in \mathscr{C}(S)$. If (iv) holds, then $\mathscr{H}=\rho k T \in \mathscr{C}(S)$ and if (v) holds, then $\mathscr{H}=\rho T k \in \mathscr{C}(S)$. Therefore each of (ii)-(v) implies (i).

Recall that $\sigma$ denotes $\omega t$, the least group congruence, and $\tau$ denotes $\epsilon K$, the greatest idempotent pure congruence.

Proposition 4.2. The following conditions are equivalent.

(i) $\operatorname{ker} \sigma=E(S)$.

(ii) For every $\rho \in \mathscr{C}(S)$, $\rho t K=\tau$.

(iii) For every $\rho \in \mathscr{C}(S), \rho K t \subseteq \tau$.

If any of these conditions hold, then also $\rho t=\rho \wedge \sigma$ for every $\rho \in \mathscr{C}(S)$.

Proof. Let $\rho$ stand for any congruence on $S$.

(i) $\rightarrow$ (ii) First observe that

$$
\begin{aligned}
& \operatorname{ker}(\rho \wedge \sigma)=\operatorname{ker} \rho \cap k e r \sigma=k e r \rho \cap E(S)=E(S), \\
& \operatorname{tr}(\rho \wedge \sigma)=\operatorname{tr} \rho \cap \operatorname{tr} \sigma=\operatorname{tr} \rho \cap \omega=\operatorname{tr} \rho,
\end{aligned}
$$

which evidently implies that $\rho t=\rho \wedge \sigma$. Since ker $\rho t=E(S)$, it follows that $\rho t \subseteq \tau$ whence $\rho t K=\tau$.

(ii) $\rightarrow$ (i). In particular, $\tau=\sigma t K=\sigma K$ so that $\sigma \subseteq \tau$ which implies that ker $\sigma=E(S)$.

(i) $\rightarrow$ (iii). By the above, $\rho K t=\rho K \wedge \sigma$ so that $k e r \rho K t=E(S)$ whence $\rho K t \subseteq \tau$.

(iii) $\rightarrow$ (i). In particular, $\omega K t=\omega t=\sigma \subseteq \tau$ and thus ker $\sigma=E(S)$.

Corollary 4.3. Let $\mathscr{K}$ be a congruence. Then the following statements are equivalent.

(i) $\operatorname{ker} \sigma=E(S)$.

(ii) For every $\rho \in \mathscr{C}(S), \rho t K=\tau$.

(iii) For every $\rho \in \mathscr{C}(S), \rho K t=\tau$. 
If any of these conditions hold, then also $\rho t=\rho \wedge \sigma, \rho K=\rho \vee \sigma$ for all $\rho \in \mathscr{C}(S)$.

Proof. From Theorem 3.6, we have that $\rho t K \subseteq \rho K t$ for any $\rho \in \mathscr{C}(S)$. Now Proposition 4.2 implies the equivalence of (i), (ii) and (iii). Let $\rho \in \mathscr{C}(S)$. By the same reference, we have $\rho t=\rho \wedge \sigma$. Also, by (i), $\epsilon \mathscr{K} \sigma$ whence $\rho=\rho \vee \epsilon \mathscr{K} \rho \vee \sigma$. Since $\operatorname{tr}(\rho \vee \sigma) \supseteq \operatorname{tr} \sigma=\omega$, it follows that $\rho K=\rho \vee \sigma$.

There is a certain amount of duality in the statements of Proposition 4.1 and Corollary 4.3. In the first

(i) $\mathscr{H}=\mathscr{H}^{*}$,

(ii) $\rho k=\rho \wedge \mathscr{H}, \quad \rho T=\rho \vee \mathscr{H}, \quad \rho k T=\rho T k=\mathscr{H}$,

(iii) $S$ has a congruence $\theta$ with $\operatorname{ker} \theta=S, \operatorname{tr} \theta=\epsilon$,

and in the second

(i) $\operatorname{ker} \sigma=E(S)$,

(ii) $\rho t=\rho \wedge \sigma, \quad \rho K=\rho \vee \sigma, \quad \rho t K=\rho K t=\tau$,

(iii) $S$ has a congruence $\theta$ with $\operatorname{ker} \theta=E(S), \operatorname{tr} \theta=\omega$,

for all $\rho \in \mathscr{C}(S)$.

\section{Completely simple semigroups}

In the discussion preceding Corollary 3.8 , we pointed out that we may restrict our attention to a sublattice $L$ of $\mathscr{C}(S)$ for which $\left.\mathscr{K}\right|_{L}$ is a congruence, and everything goes through without essential changes. On the other hand, we may preserve the setting of our main considerations in Theorem 3.6 but require $S$ to belong to some restricted class $\mathscr{C}$ of regular semigroups. As we have seen in Proposition 4.1 and Corollary 4.3, in such a case we may get certain coincidences in the diagram in Theorem 3.6 thereby the lattice corresponding to the class $\mathscr{C}$ would be a proper homomorphic image of the one depicted in Theorem 3.6.

Here we let $\mathscr{C}$ be the class of completely simple semigroups, so by Proposition 4.1, we already know that $k T=T k$. We will see in the theorem below that this is essentially the only collapse for completely simple semigroups. We make strong use of the Rees theorem by considering congruences directly on a Rees matrix semigroup of the form $S=\mathscr{H}(I, G, \Lambda ; P)$. Assuming, as we may, that the sandwich matrix $P$ is normalized, we have the following simple description of congruences on $S$.

Let $r$ and $\pi$ be partitions of $I$ and $\Lambda$, respectively, and $N$ be a normal subgroup of $G$ satisfying: for any $i, j \in I, \lambda, \mu \in \Lambda$,

$$
\text { ir } j \Rightarrow p_{\lambda i} p_{\lambda j}^{-1} \in N, \quad \lambda \pi \mu \Rightarrow p_{\lambda i} p_{\mu i}^{-1} \in N
$$


In such a case, $(r, N, \pi)$ is termed an admissible triple for $S$ and the relation $\rho=\rho_{(r, N, \pi)}$ defined by

$$
(i, g, \lambda) \rho(j, h, \mu) \Leftrightarrow i r j, g h^{-1} \in N, \lambda \pi \mu
$$

is a congruence on $S$. Conversely, every congruence on $S$ can be so represented uniquely. For related considerations and further details, consult ([1, III.4]). It will be convenient to introduce the following symbolism.

Notation 5.1. Let $S=\mathscr{M}(I, G, \Lambda ; P)$ with $P$ normalized. For every normal subgroup $N$ of $G$, define two relations $r_{N}$ and $\pi_{N}$ by

$$
\begin{array}{ll}
i r_{N} j \Leftrightarrow p_{\lambda i} p_{\lambda j}^{-1} \in N \text { for all } \lambda \in \Lambda & (i, j \in I), \\
\lambda \pi_{N} \mu \Leftrightarrow p_{\lambda i} p_{\mu i}^{-1} \in N \text { for all } i \in I & (\lambda, \mu \in \Lambda) .
\end{array}
$$

For a partition $r$ of $I$ and a partition $\pi$ of $\Lambda$, let $\overline{r \pi}$ be the normal subgroup of $G$ generated by the set

$$
\left\{p_{\lambda i} p_{\lambda j}^{-1} \mid i r j\right\} \cup\left\{p_{\lambda i} p_{\mu i}^{-1} \mid \lambda \pi \mu\right\}
$$

It follows easily that $r_{N}$ and $\pi_{N}$ are equivalence relations on $I$ and $\Lambda$, respectively.

Lemma 5.2. Let $S=\mathscr{M}(I, G, \Lambda ; P)$ and $(r, N, \pi)$ be an admissible triple for $S$. Writing triples instead of congruences and $\theta=(r, N, \pi)$, we have

$$
\begin{aligned}
\theta K & =\left(r_{N}, N, \pi_{N}\right), & \theta T & =(r, G, \pi), \\
\theta k & =(\epsilon, N, \epsilon), & \theta t & =(r, \overline{r \pi}, \pi) .
\end{aligned}
$$

Proof. All the assertions of the lemma follow easily from the definitions of an admissible triple and $\theta K, \theta k, \theta T$ and $\theta t$.

Theorem 5.3. Let $S$ be a completely simple semigroup. For $\rho \in \mathscr{C}(S)$, let $L_{\rho}$ be as in Theorem 3.6. Then $L_{\rho}$ is a homomorphic image of the lattice $L$ depicted by the following diagram 


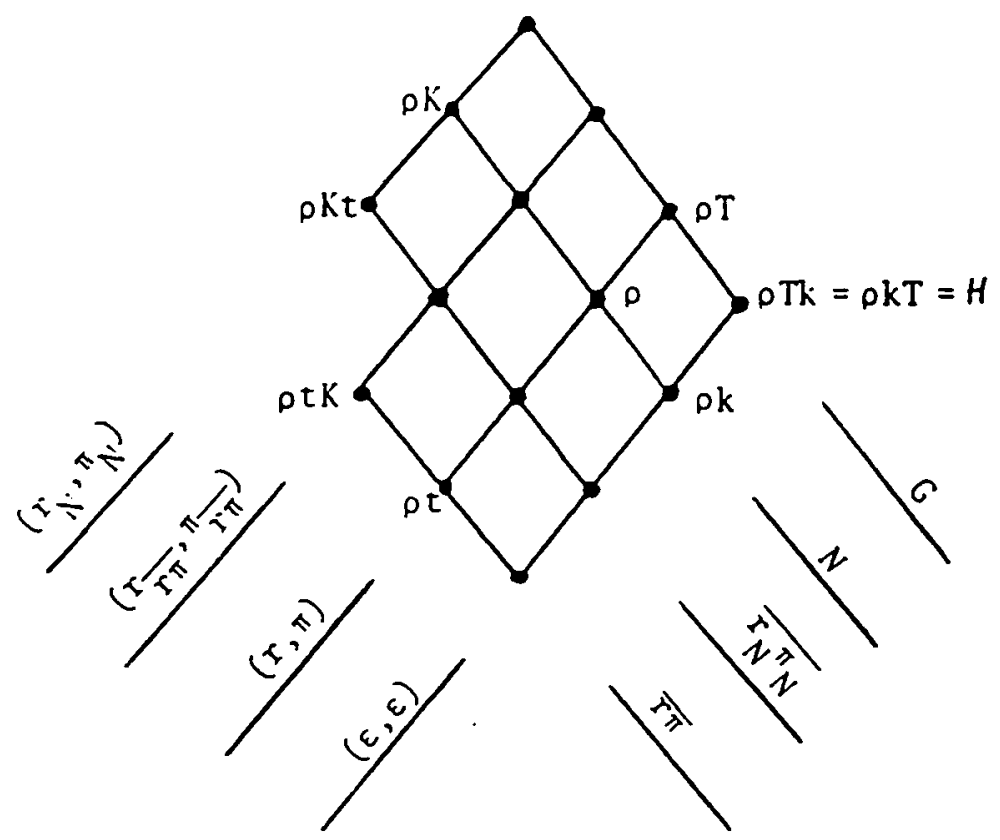

If we represent $\rho$ by an admissible triple $(r, N, \pi)$, the above labels stand for the $\mathscr{T}$-and $\mathscr{K}$-classes of the corresponding congruences. Moreover,

$$
L \cong \mathscr{F} \mathscr{D} \mathscr{L}(\rho t K, \rho K t, \rho, \rho k T) / \mathscr{R}
$$

where

$$
\mathscr{R}=(\rho t K \leq \rho K t, \rho K t \wedge \rho k T \leq \rho \leq \rho t K \vee \rho k T)
$$

and none of these relations may be omitted.

Proof. According to ([6, Lemma 3.2]), $\mathscr{K}$ is a congruence on $\mathscr{C}(S)$. Any congruence $\rho$ on $S$ can be represented by an admissible triple, say $(r, N, \pi)$. By Proposition 4.1, we know that $\rho T k=\rho k T=\mathscr{H}$. The first assertion of the theorem now follows as in the proof of Theorem 3.6. The representation of $\mathscr{T}$ - and $\mathscr{K}$-classes of the above congruences follows easily from Lemma 5.2.

Before proving the remaining assertions of the theorem, we compare it with Theorem 3.6. In fact, to the relations in Theorem 3.6 we now add the relation $\rho T k=\rho k T$. This eliminates one of the generators in Theorem 3.6, say $B=\rho T k$, the relation $\rho K t \leq \rho t K$, and we must write $\rho k T$ instead of $\rho T k$ in the third relation in Theorem 3.6. This gives the generators and relations in the present theorem.

A direct proof of the present theorem runs along the same lines as the proof of 
Theorem 3.6. For this reason, we provide only an outline, the filling in text is almost the same as there, and the notation is retained. All quadruples of 0 's and 1's satisfying the requisite conditions are listed in the following table:

\begin{tabular}{cccc}
$a$ & $A$ & $b$ & $\rho$ \\
\hline 1 & 1 & 0 & 0 \\
1 & 1 & 0 & 1 \\
\hline 0 & 0 & 1 & 0 \\
0 & 0 & 1 & 1 \\
\hline 0 & 1 & 0 & 0 \\
0 & 1 & 1 & 1
\end{tabular}.

This is used to find the lattice generated by $a, A, b, \rho$. Independence of the relations follows from the next table:

\begin{tabular}{r|llll} 
& $a$ & $A$ & $b$ & $\rho$ \\
\hline$a \leq A$ & 1 & 0 & 0 & 0 \\
$A \wedge B \leq \rho$ & 0 & 1 & 1 & 0 \\
$\rho \leq a \vee b$ & 0 & 0 & 0 & 1
\end{tabular}.

And now the example promised in Section 3.

Example 5.4. Let $S=\mathscr{M}(I, G, \Lambda ; P)$ where $I=\{1,2,3,4\}, G$ is the group of additive integers, $\Lambda=\{1,2,3\}$ and

$$
P=\left(\begin{array}{rrrr}
0 & 0 & 0 & 0 \\
0 & 1 & 5 & 13 \\
0 & 9 & 13 & 21
\end{array}\right)
$$

For $N=(2)$, we obtain

$$
r_{N}=\{\{1\},\{2,3,4\}\}, \quad \pi_{N}=\{\{1\},\{2,3\}\}, \quad \overline{r_{N} \pi_{N}}=(4),
$$

and for $r=\epsilon, \pi=\{\{1\},\{2,3\}\}$, we get

$$
\overline{r \pi}=(8), \quad r_{\overline{r \pi}}=\{\{1\},\{2\},\{3,4\}\}, \quad \pi_{\overline{r \pi}}=\{\{1\},\{2,3\}\} .
$$

It follows that $(\epsilon, N, \pi)$ is an admissible triple for $S$. For traces, we have 


$$
\begin{aligned}
& \left(r_{N}, \pi_{N}\right)=(\{\{1\},\{2,3,4\}\},\{\{1\},\{2,3\}\}), \\
& \left(r_{\bar{n}}, \pi_{\bar{r}}\right)=(\{\{1\},\{2\},\{3,4\}\},\{\{1\},\{2,3\}\}), \\
& (r, \pi)=(\epsilon,\{\{1\},\{2,3\}\}), \\
& (\epsilon, \epsilon),
\end{aligned}
$$

all of which are distinct, and for kernels

$$
G \supset N=(2) \supset \overline{r_{N} \pi_{N}}=(4) \supset \overline{r \pi}=(8),
$$

which are also distinct.

It follows that the vertices in the diagram in Proposition 5.3 are, in general, all distinct.

We can further restrict the class of semigroups under consideration by taking the class of rectangular groups, that is completely simple semigroups whose idempotents form a subsemigroup. In the normalized Rees representation $\mathscr{M}(I, G, \Lambda ; P)$ of such a semigroup, all sandwich matrix entries are equal to the identity of $G$.

Proposition 5.5. Let $S$ be a rectangular group. For $\rho \in \mathscr{C}(S)$ the sublattice of $\mathscr{C}(S)$ generated by the set

$$
\left\{\rho w \mid w \in\{K, k, T, k\}^{*}\right\}
$$

is a homomorphic image of the lattice depicted by the following diagram

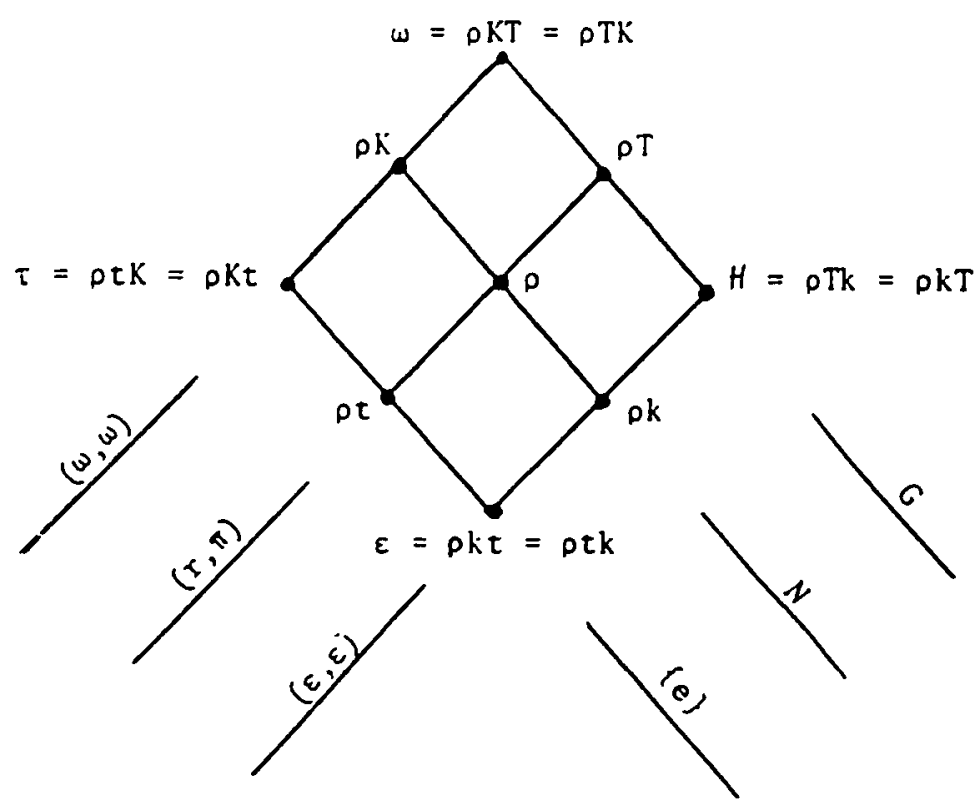


If we represent $\rho$ by an admissible triple $(r, N, \pi)$, the above labels stand for the $\mathscr{T}$ - and $\mathscr{K}$-classes of the corresponding congruences.

Proof. For any partition $r$ of $I$, any normal subgroup $N$ of $G$, and any partition $\pi$ of $\Lambda$, the triple $(r, N, \pi)$ is admissible, $r_{N}=\omega, \pi_{N}=\omega$ and $\overline{r \pi}$ is the trivial group. Let $\rho$ be represented as above. One can verify easily that $\mathscr{H}=\rho T k=\rho k T$, which also follows from Proposition 4.1, that $\tau=\rho t K=\rho K t$, which also follows from Corollary 4.3 , and that $\omega=\rho K T=\rho T K$ and $\epsilon=\rho k t=\rho t k$. In view of so much commutativity, this exhausts the elements in the set (6). The partial ordering in the diagram is clearly correct. The relations $\rho T=\rho \vee \mathscr{H}$ and $\rho k=\rho \wedge \mathscr{H}$ follow from Proposition 4.1 and $\rho K=\rho \vee \tau$ and $\rho t=\rho \wedge \tau$ from Corollary 4.3. The remaining joins and meets are well known. The argument here is similar to that in the proof of Theorem 3.6.

\section{REFERENCES}

1. J. M. HowIE, An introduction to semigroup theory (Academic Press, London, 1976).

2. F. Pastiun, The lattice of completely regular semigroup varieties, J. Austral. Math Soc A49 (1990), 24-42.

3. F. PAstiJn and M. Petrich, Congruences on a regular semigroup, Trans. Amer. Math. Soc. 295 (1986), 607-633.

4. F. Pastisn and P. G. Trotter, Lattices of completely regular semigroup varieties, Pacific J. Math. 119 (1985), 191-214.

5. M. Petrich, The kernel relation for a retract extension of Brandt semigroups, Boll. Un. Mat. Ital. B 5 (1991), 1-19.

6. M. PETriCh, The kernel relation for certain regular semigroups, Boll. Un. Mat. Ital. B 7 (1993), 87-110.

Departamento de matemática Pura

Faculdade De CrênCIAS

UNIVERSIDADE DO PORTO

P. GOMES TEIXEIRA

4050 PORTO

PORTUGAL 